\title{
Exploring the Relationship between Trunk Adiposity and Trunk Flexibility
}

\author{
TaNiqua Ward ${ }^{\mathrm{a}}$
}

\begin{abstract}
Purpose: The purpose of this study is to examine the relationship between trunk adiposity and trunk flexibility among adults. Methods: A total of 29 participants, male $(n=11)$ and female $(n=18)$ participants between the ages of 19 and 84 years. The participants were recruited from the University of Central Oklahoma daily email news service. The bioelectrical impedance analyzer (BIA) was used to calculate percent body fat and body mass index (BMI). Three circumference measurements were taken on each participant: waist, abdomen, and hips. The two inclinometers were placed on the sacroiliac joint (S1) and thoracic 12 (T12) to measure trunk flexion and extension. Trunk flexibility was measured as the difference between the two readings at full flexion or extension. Results: The Pearson's Product Moment Correlation was used to analyze the results. There was a significant negative relationship between trunk flexion correlated with abdomen circumference $(r=-.49, p=.01)$ and hip circumference $(r=-.39, p=.03)$. There was a non- significant relationship observed between trunk flexion and WHR $(r=-.10, p=$ $.62)$ and waist circumference $(r=-.35, p=.06)$. There was a non-significant relationship observed between trunk extension and waist-to-hip ratio (WHR) $(r=-.07, p=.71)$, waist circumference $(r=.17, p=.38)$, abdomen circumference $(r=.07, p=.71)$, and hip circumference $(r=.29, p=.13)$. Trunk flexion was not correlated to BMI $(r=-.27, p=.15)$ and body fat percentage $(r=-.29, p=$ .14). A significant relationship was found between trunk extension and BMI $(r=.38, p=.04)$. Trunk extension and body fat percentage $(r=.02, p=.92)$ did not have a significant relationship. Conclusion: Abdomen and hips are most beneficial when measuring circumferences for trunk adiposity. It was found that trunk flexion has a greater relationship with trunk adiposity than trunk extension.
\end{abstract}

Keywords: Flexibility, Adiposity

\section{Introduction}

Flexibility is considered an important component of physical fitness. It is defined as an individual's joints going through a range of motion (Singh N., Singh R., \& Singh S., 2011). Adiposity is considered to be the amount of fat accumulated within the body (Mark \& Janssen, 2011). This study examines both flexibility and adiposity of trunk region of the body. The purpose of this study is to examine the relationship between trunk adiposity and trunk flexibility among adults. The hypothesis of this study is that individuals with less adiposity as indicated by smaller circumferences will have a larger range of motion in the trunk region.

Very few studies have examined the relationship between trunk adiposity and trunk flexibility. Only six studies were found that observed either the combination of flexibility and exercise programs or flexibility and body composition.

This study is examining the relationship between trunk flexibility and trunk adiposity. It will closely observe circumferences and the relationship with flexion and extension. In this study the knowledge of trunk adiposity can allow an individual to focus on a larger joint such as the spine that needs to be flexible for an increasing range of motion. The trunk region of the body is an important part of flexibility because it involves bending forward, backward, and twisting motions. These motions are essential to physical fitness because it relates to the functioning of the body (Boraczyński \& Boraczyńska, 2009).

\section{Methodology}

Participants
There were a total of 29 participants, male $(n=11)$ and female $(n=18)$ participants between the ages of 19 and 84 years. The study was approved by the University of Central Oklahoma (UCO) Institutional Review Board (IRB) and all the participants completed a written informed consent. The participants were recruited through the University of Central Oklahoma daily email news service.

\section{Instruments and Procedures}

The participants had height and weight measured using the UCO digital SECA scale. Height and weight were entered into the bioelectrical impedance analyzer (BIA) to calculate percent body fat and body mass index (BMI). Three circumference measurements were taken on each participant using the Gulick tape measure: waist, abdomen, and hips. The waist measurement was taken at the smallest area of the trunk. The abdomen was measured at the naval and the hips were measured at the widest part of the bottom area of the trunk. The participant had the beginning of their sacroliliac joint located (S1). Once the S1 was located the thoracic 12 (T12) was estimated 15 centimeters $(\mathrm{cm})$ above the $\mathrm{S} 1$. The participant completed flexion first. Participants were instructed to reach down and touch their toes five times. For the sixth repetition, the two inclinometers were placed on SI and T12 and zeroed. The participant held their position reaching their toes until the measurement was taken. Extension was measured using a similar technique as flexion. The participants had to lie down on a table in a prone position. The participant was instructed to arch their back five times. On the sixth repetition, the inclinometers were placed on the two sites and zeroed. They held the position until the measurement was taken. Trunk flexibility was measured as 
the difference between the two readings at full flexion or extension.

\section{Statistical Analysis}

Statistical Package for the Social Sciences (SPSS) was used to analyze the data. Measures of trunk adiposity included waist circumference, abdominal circumference, hip circumference, and WHR. Total body composition was also assessed as BMI and percent body fat. Two measures of trunk flexibility were measured: trunk flexion and trunk extension. The Pearson's Product Moment Correlation was used to examine relationships between measures of trunk adiposity and trunk flexibility as well as relationships between total body composition and trunk flexibility.

\section{Literature Review}

According to Boraczyński and Boraczyńska (2009) physical activity can have an impact on flexibility. This study examined the different types of physical activity and how it can influence flexibility. The two different physical activity methods examined were physical education $(n=235)$ and physiotherapy $(n=142)$. Flexibility was tested by the sit-andreach test. Prior to completing the test the students had their body composition tested by a Tanita BC 418 MA body composition analyzer. The results indicated that the students in the physical education were within normal range for body mass and body fat, whereas the students in the physiotherapy were overweight. The students in the physical education (6.4 $\mathrm{cm}$ and $6.3 \mathrm{~cm}$ in men and women) had significantly higher flexibility levels $(p<0.001)$ on the sit-and-reach test than the physiotherapy students. It was concluded that the greater amount of physical activity a student has the higher level of physical fitness and flexibility will be demonstrated.

A similar study conducted by Sekendiz, Altun, Korkusuz, and Akin (2007) examined the impact of Pilates on trunk strength, endurance, and flexibility. A total of 38 women participated in the study. There were 21 women that were part of the Pilates exercise group and 17 women part of the control group. The Pilates group had a total of 15 sessions, three sessions a week for five consecutive weeks. Both the Pilates group and the control group were given pre and post testing on the variables measured. Posterior trunk flexibility was measured doing the sit-and- reach test. Body fat was measured using skin-fold thickness. BMI was calculated from weight and height. The results were analyzed using an independent sample t-test to examine the significant difference between the pre and post testing in the Pilates and control group. The results indicated that there was no difference between the two groups in BMI and body fat percentage. However, there was a significant difference $(p<0.05)$ between the two groups in posterior trunk flexibility. The author concluded that Pilates exercises were effective at improving trunk flexibility.

Another study was conducted that examined body composition and flexibility; however, it categorized the participants into groups based on health status. The study that observed the relationship between the components of nutritional status, waist circumference, physical activity, and flexibility in boys was performed by Fernandes et al. (2007). A total of 74 boys participated in the study. The boys had height and weight measured and then used the BIA to compute the percentage of body fat. The percentage was used for nutritional status and to categorize the boys: well nourished (G1), overweight (G2), and obese (G3). Physical activity was measured by the boys completing the International Physical Activity Questionnaire (IPAQ). Flexibility was measured using the sit and reach test. The sit and reach then classified the boys as "fit" or "unfit" based on age and gender. Waist circumference was measured using an anthropometric tape measure. The data was analyzed by the analysis of variance (ANOVA) for the differences in nutritional statuses. The results indicated that the larger waist circumferences were found in group G3. According to body fat levels and flexibility using the sit and reach there was a significant difference $(p=0.048)$ between G1 and G3. G1 were able to produce the highest levels of flexibility. G2 did not have a significant difference from G1 and G3. The results also found that there was a significant correlation in G3 with body fat and sit and reach $(p=0.025)$. Physical activity levels and sit and reach were found to have significant correlations in G1 $(p=0.022)$. In conclusion there is not a difference between the well nourished boys (G1) and the overweight boys (G2). However, the obese boys (G3) were found to have their flexibility levels impacted due to weight and body composition. Body fat percentage was found to have an influence on the flexibility performing the sit-and-reach test. While this study examined male's body composition and flexibility, another study examined females.

Pasbakhsh, Ghanbarzadeh, and Ebadi (2011) examined female students and their relationship between skinfold, BMI, and physical activity. A total of 308 females between the ages of 11-13 were randomly selected to participate in the study. Skinfolds were taken at three sites: triceps, quadriceps, and suprailiac. Skinfolds were used to calculate the percentage of body fat. The students were then divided into three groups based on percentage of body fat. The groups were categorized as low, medium, and high. BMI was calculated from height and weight. Physical activity was measured with American Alliance for Health, Physical Education, and Recreation (AAHPER) tests that included performing sit ups with the bar, sit ups, flexibility with the sit and reach, and running. Pearson's correlation was used to analyze the results. Based on the physical fitness tests and body fat percentage there was a significant correlation $(p<0.001)$. When results were being examined it was illustrated that there was an inverse relationship between flexibility and percentage of body fat. Flexibility and percentage body fat had a significant weak negative correlation $(r=-0.244, p<0.01)$. The author concluded that the percentages of body fat and physical fitness have an inverse relationship.

A study that was found by Li-ming et al. (2008) observed anthropometric indices as a predictor of trunk obesity in Chinese adults. The purpose of the study was to find the best method of measuring trunk obesity. A total of 1,946 adults aged 20-40 years old participated in the study (853 women and 1,093 men). The participants had height and weight measured which was then used to calculate BMI. Three waist and hip circumferences were measured using an anthropometric tape at the narrowest part of the waist, the iliac crest, and the maximum part of the buttocks. Waist-tohip ratio (WHR) was calculated using the measurements. Total fat mass was measured by using the dual $\mathrm{x}$-ray absorptiometry scan (DXA). The conicity index ( $C$ index) was calculated from waist circumference. The results 
indicated that men and women's total fat mass was significantly correlated $(p<0.0001)$ to BMI $(r=0.85$ and $r=0.76$ in men and women), waist circumference ( $r=0.80$ and $r=0.72$ in men and women), WHR ( $r=0.45$ and $r=0.40$ in men and women), and $C$ index ( $r=0.45$ and $r=0.34$ in men and women). It was observed that BMI and waist circumference were better predictors of trunk obesity in Chinese adults than WHR and $C$ index. While this study examined a demographic population for trunk adiposity, the following study examined athlete's trunk adiposity and flexibility.

Singh N., Singh R., and Singh S., (2011) conducted a study that examined trunk flexibility and body composition of athletes. Fifty university football $(n=25)$ and badminton $(n=25)$ players completed the sit-and-reach and skinfold thickness assessments. Once the data was collected software was used to find results by using the one tailed t-test with a significance $(p=0.05)$. The results indicated that there was no significant difference in football and badminton players flexibility $(t=0.321)$. There was also no significant difference found in the percentage body fat $(t=0.09)$ and total body fat $(t=1.005)$ between the two groups. Although all the raw scores of the football players were slightly higher than the badminton players it did not influence flexibility between the two athletes.

There have been few studies conducted on trunk flexibility and trunk adiposity. The studies that were observed concluded that there is a relationship between adiposity and flexibility of the trunk region. Many of the individuals in the studies that had a higher BMI or body fat percentage were not able to perform flexibility as well as the individuals with lower percentages. There needs to be more research on the relationship between trunk flexibility and adiposity. The knowledge of appropriate circumference and weight can help individuals increase range of motion.

\section{Results}

The descriptive statistics for trunk flexibility, trunk adiposity, and total body composition are presented in Table 1. The Pearson's Product Moment Correlation values are shown in Table 2.

Relationships between trunk flexion and measures of trunk adiposity were examined. There was a non-significant relationship observed between trunk flexion and WHR ( $r=-$ $.10, p=.62)$ and waist circumference $(r=-.35, p=.06)$. On the contrary, there were significant moderate negative relationships between trunk flexion and the circumference of the abdomen $(r=-.49, p=.01)$ and hips $(r=-.39, p=.03)$.

In addition, correlations between trunk extension and measures of trunk adiposity were examined. A non-significant relationship was observed between trunk extension and WHR ( $r=-.07, p=.71$ ), waist circumference $(r=.17, p=.38)$, abdomen circumference $(r=.07, \quad p=.71)$, and hip circumference $(r=.29, p=.13)$. Relationships between trunk flexibility and measures of total body composition were also analyzed. Trunk flexion was not significantly correlated with BMI $(r=-.27, p=.15)$ or body fat percentage $(r=-.29, p=.14)$. When correlations between trunk extension and BMI were examined, a significant moderate positive relationship was found ( $r=.38, p=.04$ ). Trunk extension and body fat percentage $(r=.02, p=.92)$ did not have a significant relationship.

Age of the participants was an additional variable that was examined. Age had a significant strong negative relationship with trunk flexion $(r=-.59, p=.001)$. There was a non-significant negative relationship between age and trunk extension $(r=-.29, p=.12)$.

Table 1

\begin{tabular}{|c|c|c|c|c|}
\hline & MEAN & SD & MIN & MAX \\
\hline Age (years) & 39.70 & 24.86 & 19 & 84 \\
\hline Waist Circumference (cm) & 78.79 & 12.36 & 59 & 104 \\
\hline Abdominal Circumference $(\mathbf{c m})$ & 84.49 & 14.87 & 59 & 114 \\
\hline Hip Circumference (cm) & 99.60 & 11.68 & 79 & 125 \\
\hline Waist-to-hip ratio & 0.79 & 0.06 & 0.67 & 0.92 \\
\hline Body Mass Index $\left(\mathrm{kg} / \mathrm{m}^{2}\right)$ & 26.05 & 6.84 & 15 & 45.10 \\
\hline Body Fat (percentage) & 27.45 & 13.21 & 4.80 & 49.80 \\
\hline Flexion (degree) & 49.56 & 14.55 & 22.00 & 78.00 \\
\hline Extension (degree) & 12.98 & 12.38 & -2.00 & 50.00 \\
\hline
\end{tabular}

Table 2 
Pearson's Product Moment Correlation Values

\begin{tabular}{|c|c|c|c|c|c|c|c|c|c|}
\hline & AGE & WST & $\overline{\mathbf{A B}}$ & HIP & WHR & $\overline{\text { BMI }}$ & $\overline{\mathbf{B F}}$ & $\overline{F L}$ & $\overline{\mathbf{E X}}$ \\
\hline AGE & & $.37 *$ & $.63^{* *}$ & $.38^{*}$ & .16 & .19 & $.68^{*}$ & $-.59 *$ & -.29 \\
\hline WST & & & $.88^{* *}$ & $.86^{* *}$ & $.66 * *$ & $.81 * *$ & $.39 * *$ & -.35 & .17 \\
\hline$\overline{\mathbf{A B}}$ & & & & $.89 * *$ & $.40^{*}$ & $.75^{* *}$ & $.67 *$ & $-.49 * *$ & .07 \\
\hline HIP & & & & & .20 & $.89 * *$ & $.54 * *$ & $-.39 *$ & .29 \\
\hline WHR & & & & & & .25 & .03 & -.10 & -.07 \\
\hline BMI & & & & & & 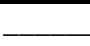 & $-.52 * *$ & -.27 & $.38 *$ \\
\hline $\mathbf{B F}$ & & & & & & & & -.29 & .02 \\
\hline FL & & & & & & & & 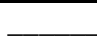 & .18 \\
\hline EX & & & & & & & & & \\
\hline
\end{tabular}

Note. . Waist $=$ WST, Abdomen=AB, Waist-to-hip ratio=WHR, Body Mass Index=BMI, Body Fat=BF, Flexion=FL,

Extension $=\mathrm{EX}$

* Correlation is significant at the 0.05 level

** Correlation is significant at the 0.01 level

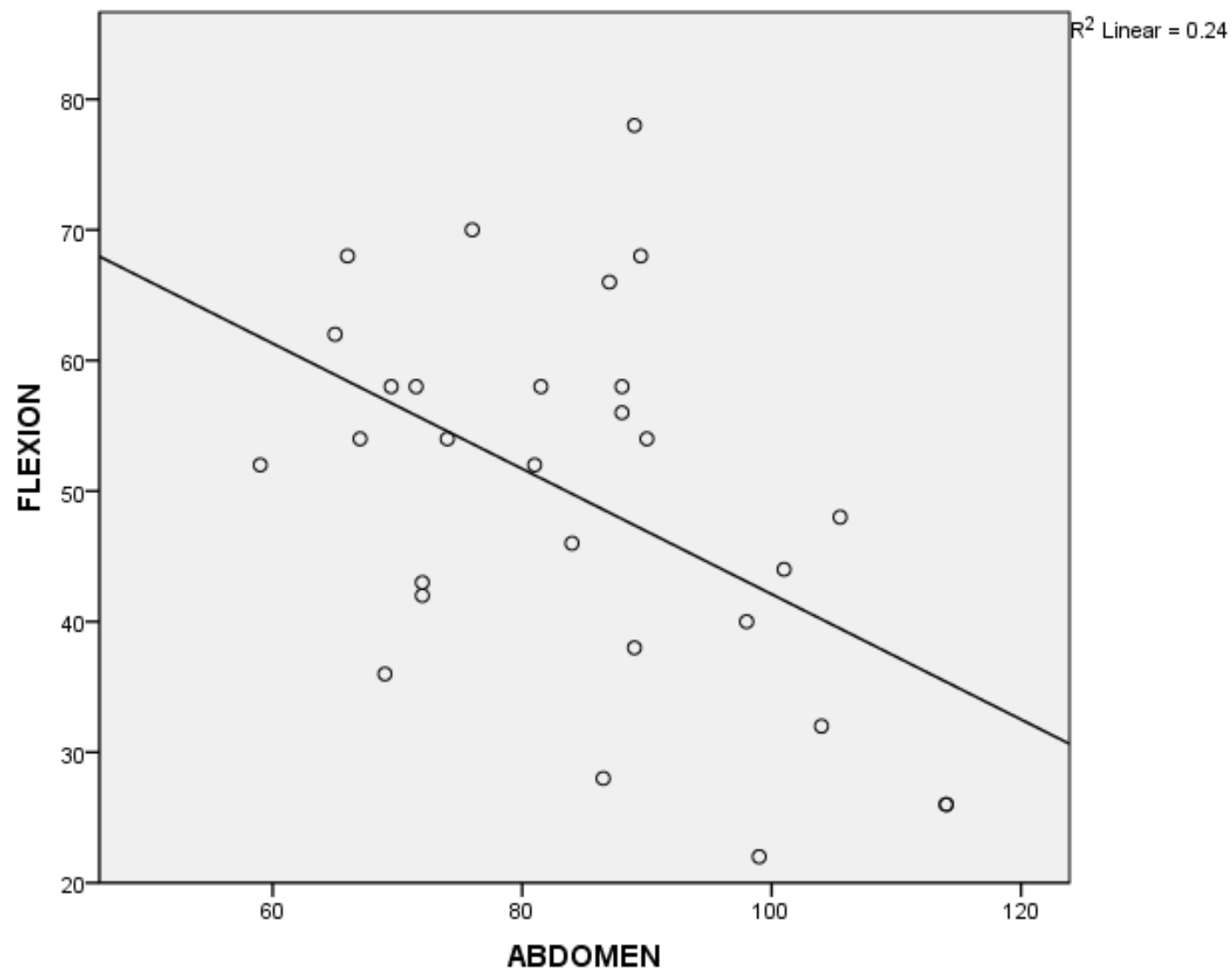

Figure 1. The scatter plot illustrates that the range of trunk flexion and abdomen circumference. There was a significant moderate negative relationship $\left(r^{2}=.24, p=.01\right)$ between trunk flexion and abdomen circumference. 


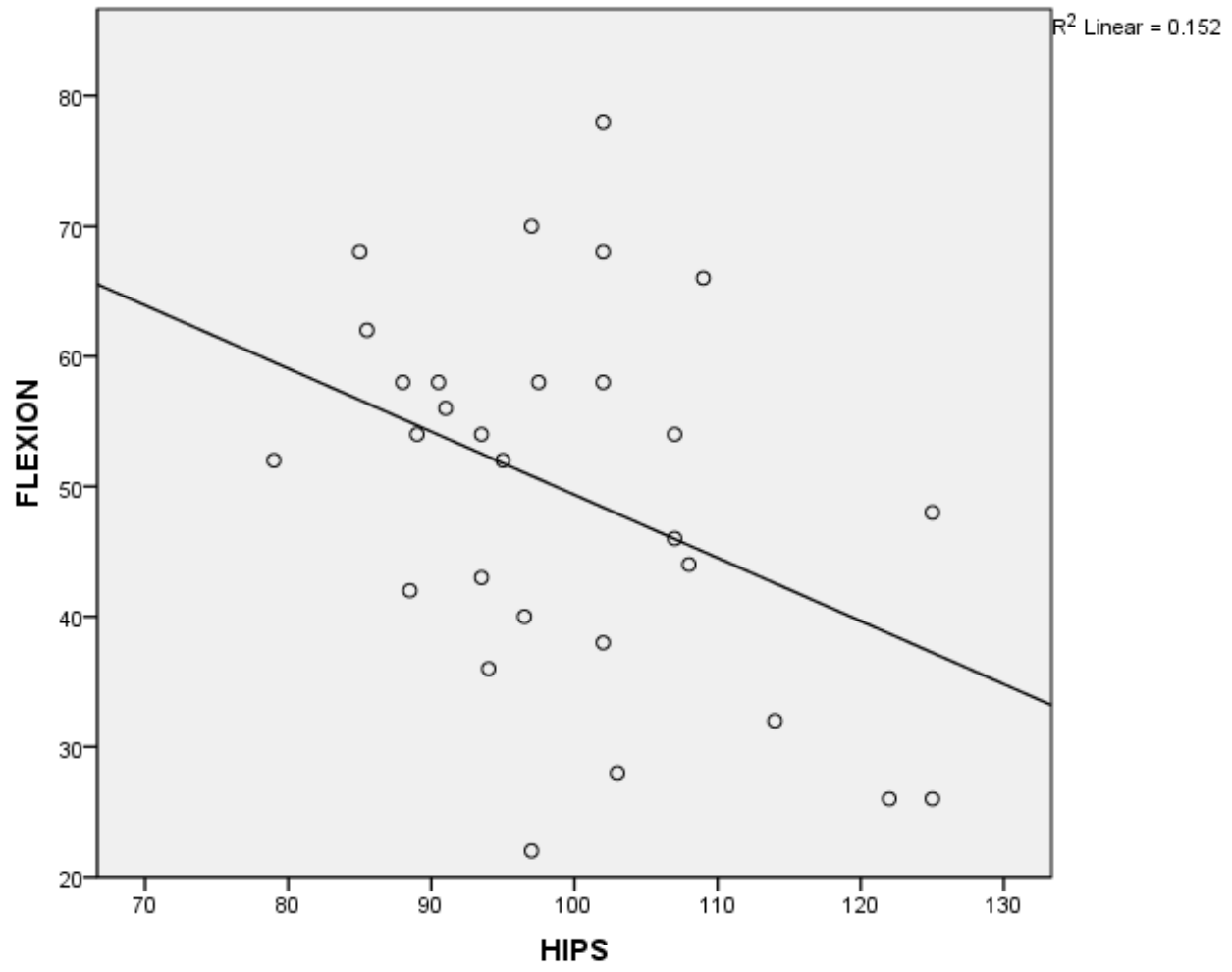

Figure 2. The scatter plot illustrates the relationship between trunk flexion and hip circumference. There was a significant moderate negative relationship $\left(r^{2}=.152, p=.03\right)$ between trunk flexion and hip circumference.

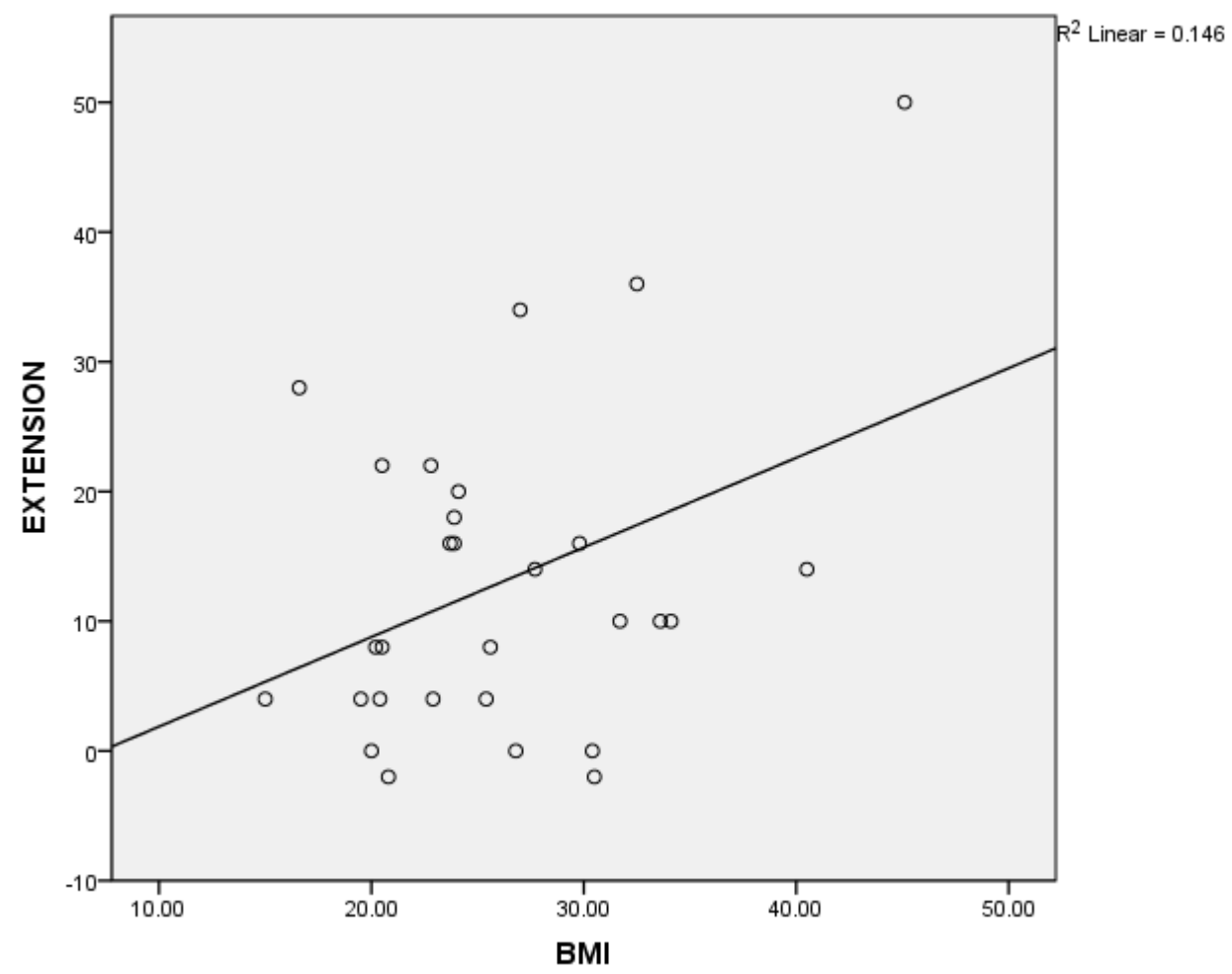

Figure 3. The scatter plot illustrates the range of trunk extension and BMI. There was a significant moderate positive relationship $\left(r^{2}=.146, p=.04\right)$ between trunk extension and BMI. 


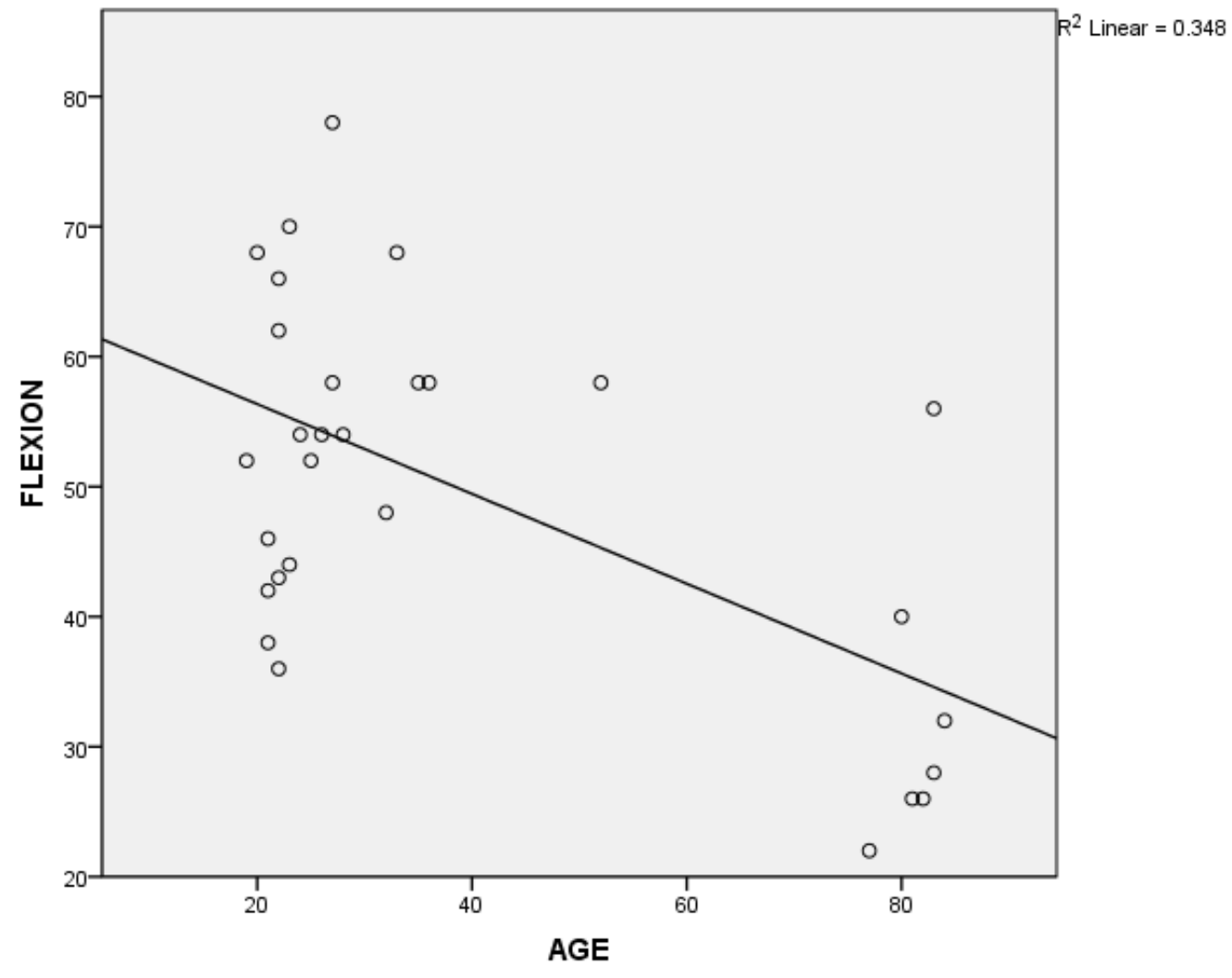

Figure 4. The scatter plot illustrates the range of trunk flexion and age. There was a significant strong negative relationship $\left(r^{2}=\right.$ $.348, p=.001$ ) between trunk flexion and age.

\section{Discussion}

Trunk flexibility and trunk adiposity were found to have relationships indicated by the correlation values. The primary questions of this study examined the relationships between measures of trunk adiposity and trunk flexibility. The results of this study showed that some measures of trunk adiposity are related to trunk flexion. The participants that had less trunk adiposity were able to perform trunk flexion better than those with larger amounts of trunk adiposity.

\section{Trunk Flexibility and Trunk Adiposity}

The only significant correlations were found between trunk flexion and circumferences. The results that were found indicated that there was a significant moderate negative relationship between abdomen circumference $(r=-.49, p=.01)$ and hip circumference $(r=-.39, p=.03)$. The lower the circumference of the abdomen and hip the larger range of motion the individual had in trunk flexion. The relationships found between circumferences and trunk extensions were | found to be non--significant. This indicates that trunk flexion may have a greater relationship with trunk adiposity than trunk extension.

Trunk flexion having a greater relationship with trunk adiposity may be due to the placement of the fat storage. Fat is being stored in the abdominal area which is in front of the body. The larger the amount of fat in front makes it more difficult to have a larger range of motion to do flexion which is performed by bending forward. Trunk extension may not have as great of a relationship because there is limited variability in trunk extension and the storage placement of where fat is stored. There have not been many changes seen in trunk extension and trunk adiposity. It is more difficult to find relationships between trunk extension and trunk adiposity variables.

\section{Trunk Flexibility and Total Body Composition}

The relationship between trunk flexibility and total body composition was examined as a secondary question. The only significant relationship found was between trunk extension and BMI ( $r=.38, p=.04)$. The components height and weight are measured to calculate BMI. The relationship between trunk adiposity and BMI can also be related to the individual's health status. Individuals with greater BMI levels are more likely to be overweight or obese. Those individuals with greater BMI levels possibly store more fat than those that have lower BMI levels (Fernandes et al., 2007). This generalization does have an exception for some individuals. The use of BMI as an indicator of body fat is not always accurate for children or athletes.

The finding that trunk extension is positively correlated with BMI is surprising. Most of the findings are related to trunk flexion and adiposity. Majority of testing that has been conducted measures trunk flexion, few studies measure trunk extension. There have not been many research studies on trunk flexibility and BMI. However, studies do suggest that 
body composition is related to trunk flexibility instead of trunk extension.

\section{The Impact of Age}

Although age was not a primary variable measured in this study, it may be helpful in understanding the relationships that were observed. There was a wide age range of participants in this study from 19-84 years. Age can influence flexibility in many ways. Older adults have a higher body fat percentage than younger adults due to sarcopenia, which is the loss of muscle mass due to age (Puthoof \& Neilsen, 2007). The decline in strength and power gradually causes functional limitations in older adults. Also, as adults age they can have a decreased range of motion (Bell \& Hoshizaki, 1981). Older adults had lower levels of trunk flexion and extension when compared to younger adults which is indicated in Figure 4. Other factors that influence flexibility include gender of individuals. Females tend to have greater flexibility than males. Also, the lower extremity joints tend to decline at a faster rate than upper extremity joints. There was not a relationship between trunk extension and age due to the limited variability in trunk extension. However, there was a significant strong negative relationship between flexion and age $(r=-.59, p=.001)$.

\section{Conclusion}

Based on this study trunk flexion has a negative moderate relationship with adiposity. The circumference of the abdomen and hips appear to be most closely related to trunk flexion. Adults need flexibility because it is an important component of functional fitness (Klein, Stone, Phillips, Gangi, \& Hartman, 2002). Stretching can help increase flexibility in joints. All the major muscle groups need to be stretched once a day as recommended by American College of Sports Medicine (Pollock et al., 1998). It was been found through other research that increasing flexibility is most beneficial when combining stretching with an exercise regimen (Cavani, Mier, Musto, \& Tummers, 2002). This can improve range of motion while also targeting physical activity.

\section{References}

Bell, R. D., \& Hoshizaki, T. B. (1981). Relationships of age and sex with range of motion of seventeen joint actions in humans. Canadian Journal of Applied Sport Sciences, 6(4), 202-206. Retrieved from http://www.nrcresearchpress.com

Boraczyński, T., \& Boraczyńska, L. (2009). The influence of physical activity on body composition and the level of student's flexibility. Medicina Sportiva, 13(1), 13-16. doi:10.2478/v10036-009-0003-8.

Cavani, V. V., Mier, C. M., Musto, A. A., \& Tummers, N. N. (2002). Effects of a 6-week resistance-training program on functional fitness of older adults. Journal of Aging \& Physical Activity, 10(4), 443452. Retrieved from http://journals.humankinetics.com/japa

Fernandes, R., Christofaro, D., Cucato, G., Agostini, L., de Oliveira, A., \& Júnior, I. (2007). Nutritional status, physical activity level, waist circumference, and flexibility in brazilian boys. Brazilian Journal of Kineanthropometry \& Human Performance, 9(4), 321-326. Retrieved from http://www.periodicos.ufsc.br/index.php/rbcdh/

Klein, D. A., Stone, W. J., Phillips, W. T., Gangi, J. J., \& Hartman, S. S. (2002). PNF training and physical function in assisted-living older adults. Journal of Aging \& Physical Activity, 10(4), 476-488. Retrieved from journals.humankinetics.com/japa

Li-ming, L., Shu-feng, L., Xiang-ding, C., Fei-yan, D., Li-jun, T., Xue-zhen, Z., \& Hong-wen, D. (2008). Anthropometric indices as the predictors of trunk obesity in chinese young adults: receiver operating characteristic analyses. Annals of Human Biology, 35(3), 342-348. doi:10.1080/03014460802027049.

Mark, A. E., \& Janssen, I. (2011). Influence of movement intensity and physical activity on adiposity in youth. Journal of Physical Activity \& Health, 8(2), 164173. Retrieved from http:// humankinetics.com

Pasbakhsh, H., Ghanbarzadeh, M., \& Ebadi, G. (2011) Relationships between skinfold thickness, body mass index and physical fitness of female students. Studies in Physical Culture \& Tourism, 18(2), 149155. Retrieved from http://www.awf.poznan.pl

Pollock, M. L., Gaesser, G. A., Butcher, J. D., Despres, J. P., Dishman, R. K., Franklin, B. A., \& Ewing Garber, C. C. (1998). American college of sports medicine position stand: the recommended quantity and quality of exercise for developing and maintaining cardiorespiratory and muscular fitness, and flexibility in healthy adults. Medicine \& Science in Sports \& Exercise, 30(6), 975-991. Retrieved from http://www.mhhe.com

Puthoff, M., \& Nielsen, D. (2007). Relationships among impairments in lower-extremity strength and power, functional limitations, and disability in older adults. Physical Therapy, 87(10), 1334-1347. Retrieved from http://ptjournal.apta.org/content

Sekendiz, B., Altun, O., Korkusuz, F., \& Akin, S. (2007). Effects of Pilates exercise on trunk strength, endurance and flexibility in sedentary adult females. Journal of Bodywork \& Movement Therapies, 11(4), 318-326. doi:10.1016/j.bmt.200.12.002.

Singh, N., Singh, R., \& Singh, S. (2011). Study of trunk flexibility and body composition between football and badminton players. Journal of Physical Education \& Sport, 11(1), 18-21. Retrieved from http://efsupit.ro 